\title{
Cherenkov differential detector at the Yakutsk extensive air shower array
}

\author{
Egorov Yuriy Alexandrovich ${ }^{1}$ \\ Y.G. Shafer Institute of Cosmophysical Research and Aeronomy \\ Russia, Yakutsk
}

zeppelineikfia.ysn.ru

\section{Petrov Zim Egorovich}

Y.G. Shafer Institute of Cosmophysical Research and Aeronomy

Russia, Yakutsk

pzelikfia.ysn.ru

\section{Knurenko Stanislav Petrovich}

Y.G. Shafer Institute of Cosmophysical Research and Aeronomy

Russia, Yakutsk

Knurenko@ikfia.ysn.ru

Abstract: A new instrument for investigating Cherenkov-Vavilov radiation on the basis of a camera Obscura, called for short the Cherenkov differential detector (CDD), was introduced at the Yakutsk Extensive Air Shower Array in 2003.

The Cherenkov differential detectors are located around the center of the current Yakutsk EAS array. This location allows the detectors to operate as part of the larger array and use its data: trigger signal, shower arrival angles, axis coordinates in the installation plane, etc. The CDD photomosaic consists of a photomultiplier array, each of the photomultipliers observing a certain height of the celestial sphere through a narrow slit of the camera. An important advantage of CDD is the ability to determine the spatio-temporal pattern of the development of the Cherenkov component of the EAS in the atmosphere. 


\section{Introduction}

Synchronous measurements of the flow of charged particles on the installation plane by a large number of scintillator and Cherenkov-Vavilov radiation [1-3 ]detectors provide an integral picture of the shower development, i.e. the total number of electrons, muons, and gamma quanta. Recording the detector response on the arrival time of individual groups of particles enables us to reconstruct the spatiotemporal picture of the shower development in the atmosphere and better understand the pattern of nuclear interactions at ultrahigh energies [4-5]

At the Yakutsk EAS Array [6], the B. Khrenov group's [7] idea has been realized. At this stage of the experiment, three Cherenkov differential detectors are operating at the Yakutsk EAS Array, designated by us as Obscura1, 2, 3. (Fig. 1)

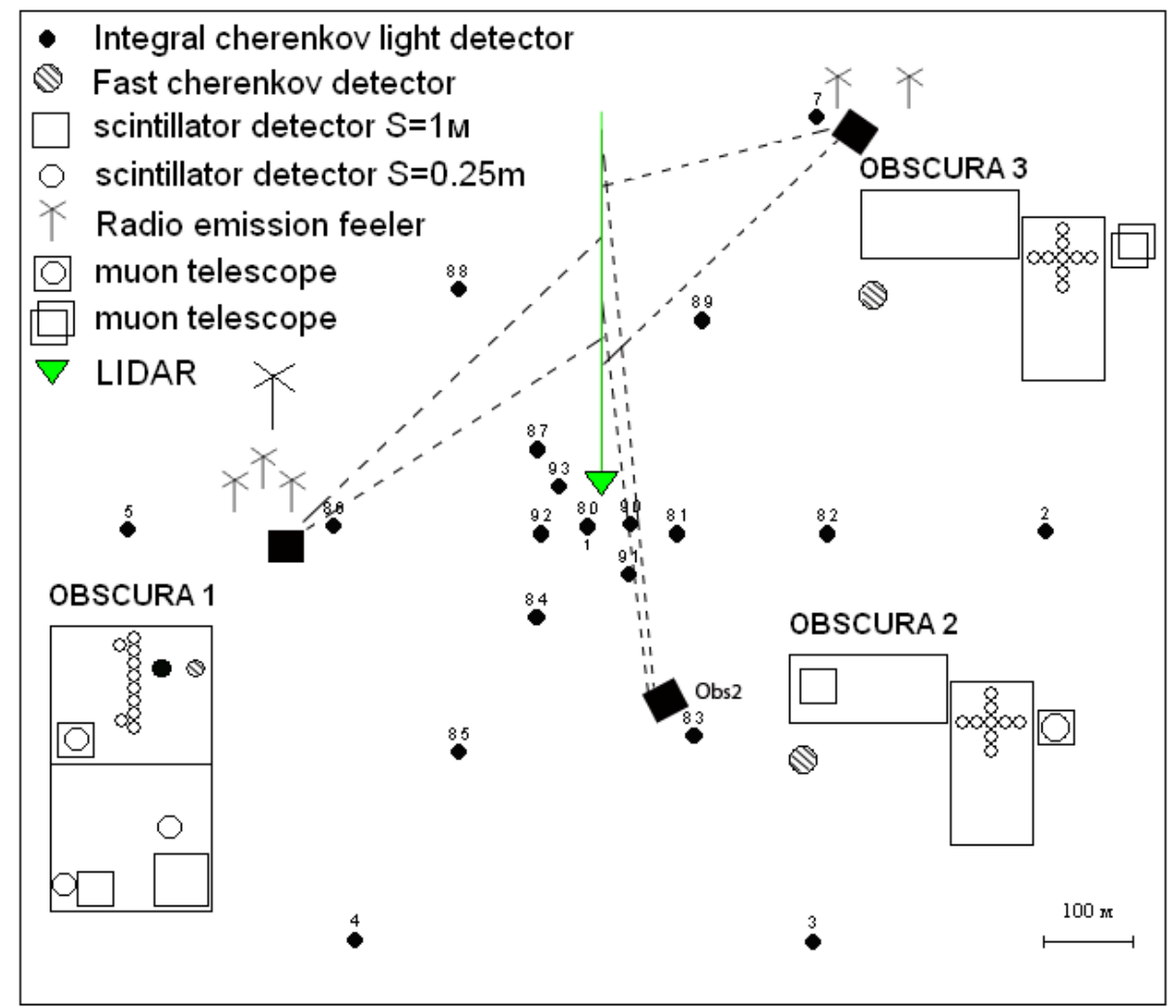

Figure 1: Diagram of the Cherenkov part of the Yakutsk EAS Array

The Cherenkov differential detectors (CDD) are located at a distance of 250-500 m from the center of the current Yakutsk EAS Array. This location allows the detectors to work as part of the array and record combined showers. The localization of the shower axis in space (the definition of $\theta, \varphi$ and the coordinates of the intersection of the axis with the installation plane) is performed according to the data of the current larger installation. One of the advantages of CDD in comparison with Cherenkov integral detectors is the substantial suppression of the noise background of the night sky. A low noise level is achieved by selecting a small field of view through the slit for each photomosaic photomultiplier. The registration of Cherenkov light with the CDD increases the observation time of shower events, since the detector can operate at dusk and on moonlit nights.

\section{Obscura 1 Installation}

The camera obscura-based Cherenkov differential detector consists of two parts: camera obscura (room), photomosaic and data collection system. The camera is a light-tight room 
with darkened walls. (Fig. 2) On the room ceiling, which is a strictly horizontal plane, two parallel slits are cut perpendicular to the photomosaic axis (Obscura 1 and Obscura 3). Obscura 2 is equipped with a circular slit.

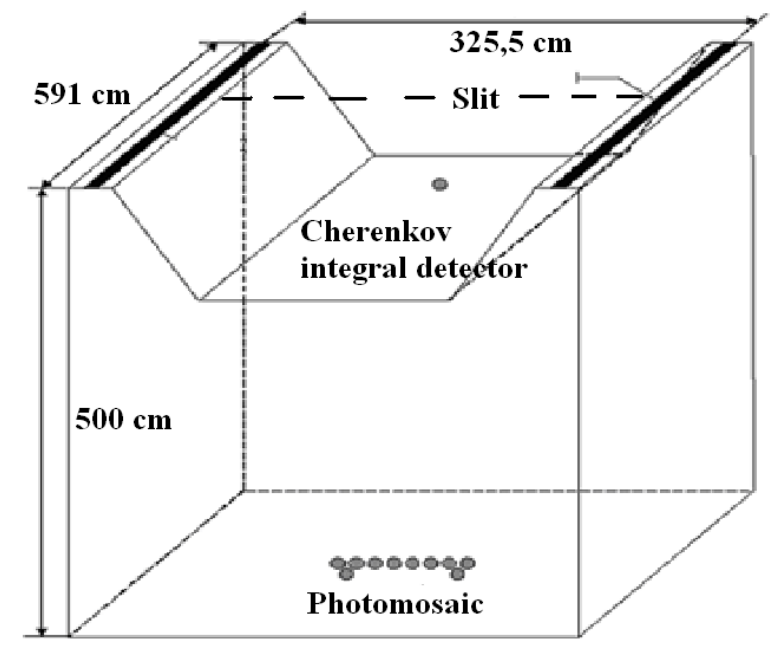

Figure 2: Camera Obscura 1

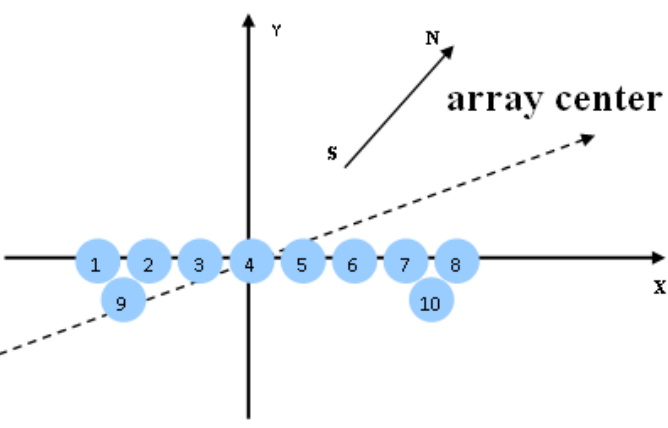

Figure 3: Photomosaic

Nearer slit (to the installation center) slit: length $-591 \mathrm{~cm}$, width $-8.7 \mathrm{~cm}$; Farther slit: length $-591 \mathrm{~cm}$, width $-8.3 \mathrm{~cm}$.

The distance between the slit axes (lines passing along the slits in the middle) is $325.5 \mathrm{~cm}$. The Cerenkov integral detector is located outside between the slits.

The photomosaic consists of 10 photomultipliers placed along a line perpendicular to both slits and at the same distance from the ends of each slit, respectively. (Fig. 3)

The photocathodes of all photomultipliers are located on the same plane, which is strictly parallel to the plane of the slits and forms a right angle with the vertical.

The distance between the planes of the slits and the photomultiplier photocathodes is $515 \mathrm{~cm}$. The geometric center of the pinhole camera obscura is located in the middle of the photomultiplier photocathode of Photomosaic No. 4 (Fig. 3). The photomultiplier direction is 55.5 degrees east of the direction to the north.

In the photomosaic plane, the image of EAS Cherenkov radiation is manifested as a sequence of responses of the emitted photomultipliers with different amplitudes and shapes. The level of the amplitudes recorded in the image depends on the energy of the primary EAS particle and the shower trajectory relative to the camera slits, i.e. coordinates of the shower axis in the installation plane, the zenith angle $\theta$, and the azimuth $\varphi$ angles.

In addition to Cerenkov detectors, 5 ground scintillation detectors of a charged component and one underground detector (with a threshold of $1.5 \mathrm{GeV}$ ) are operating at the facility recording muons

\section{Small Cherenkov installation.}

The small Cherenkov installation (SCI) is an independent Cherenkov instrument that simultaneously registers signals from Cherenkov condensation stations in the center of a larger installation. [6] On the basis of these detectors and an additional recorder, a small Cherenkov installation is formed which selects events in the region of $10^{15}-10^{17} \mathrm{eV}$. The SCI recorder is made according to the CAMAC standard, it has its own registers that allow processing input signals from the connected detectors and generate its own trigger - a "Small Master" for recording the event frame on the CDD. To control the CAMAC system, a separate personal computer is used, which accumulates data on small showers and control information, independently of the main Central Recorder. In order to determine the direction of showers 
arrival in the SCI, special electronics was added in the Cherenkov radiation detector channel, allowing the station to generate a fast signal and transmit it on the same cable before the pack from the LC converter to the additional recorder, which is where temporary measurements are conducted with a resolution of $20 \mathrm{MHz}$.

The observation session consists of several 15 minute parts, between which the background frequency of the detectors is monitored and a separate program block instructs the LIDAR laser calibration pulse to start.

In addition, in parallel with the recording of events, the amplitude spectra of the detectors are taken with the production of a separate spectra file.

\section{Data collection system}

To register the PMT response form, computers of the Pentium enhanced reliability class are used with a backplane for PCI slots. PCI slots are equipped with La-n10m8 PCI cards with two fast 8-bit ADCs with a sampling frequency of $100 \mathrm{MHz}$ and a buffer memory of $2 \mathrm{MB}$. The system is equipped with the software control of the amplification of signals from the PMT outputs, each board serving 2 measurement channels. The observation points are integrated into the overall installation network. Data transmission and time synchronization of stations are carried out over the fiber-optic network (Fig. 4).

The registration control is performed from the external master signals. To generate a "Master" from the larger installation, it is required that the responses from three scintillation detectors coincide (the distance between the detectors is $500 \mathrm{~m}$ ) not lying on the same straight line. "Masters" of the small Cherenkov installation [8-9], require the coincidence of responses from three Cherenkov integral detectors located at the vertices of equilateral triangles with sides of 50,100 and $250 \mathrm{~m}$. The frequency of such events is about 30 times per hour. This selection system makes it possible to record pulses in showers with energies from $10^{15}$ to $10^{17} \mathrm{eV}$.

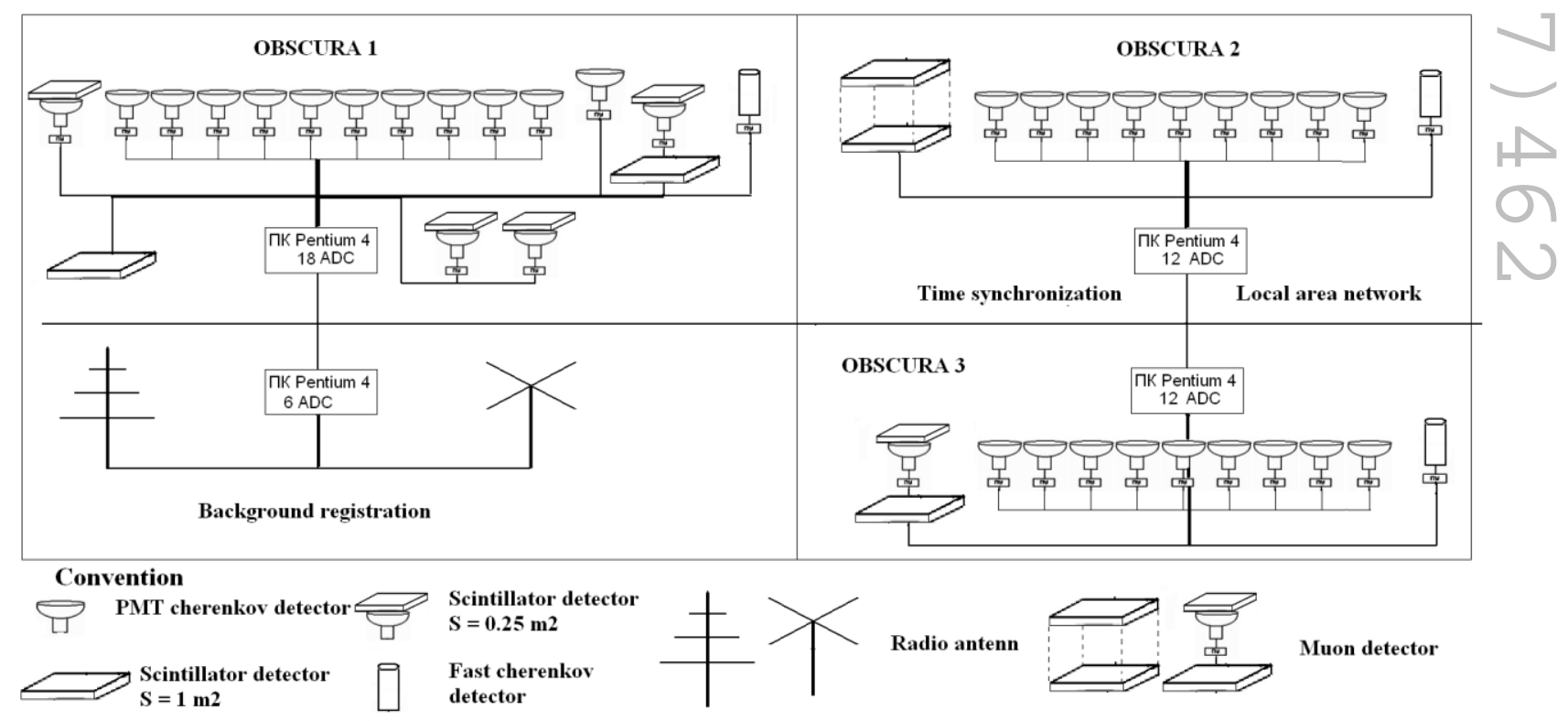

Figure 4: Block diagram of the CDD registration system

The registration programs start the ADC in a continuous mode and convert the signals from the PMT outputs and cyclically record them into the buffer memory area called the "prehistory". The "prehistory" contains the records of ADC samples for the last $13 \mu$ s of signals, the time interval between neighboring samples being $10 \mathrm{~ns}$. After the arrival of "masters" from the large installation or SCI, the area of the buffer memory called "history" records color signals of the "Master" type and data on the amplitude of the calibration LED, forming, in combination 
with the prehistory, an event frame (Fig. 5). The received frames of shower events are made into a file record and added to the initial file created automatically at the start of the observation session.

During the daylight hours, the installation only records the responses of light-tight scintillation detectors.

Thus, the CDD provides the collection and accumulation of observational data sufficient for analyzing the spatiotemporal characteristics of shower events.

\section{Software}

The CDD software includes 3 components: recording of observations, preliminary analysis and recording of calibration measurements with their processing. All software components work in a Windows environment and have a graphical interface for controlling the operation of programs.

The CDD detectors are divided into 3 groups: photomosaic (from 9 to 10 photomultipliers), an integrated detector and scintillation detectors (from 5 to 8 detectors). Prior to the observation session, the gain of the ADC boards common for each group is set, after which the registration program goes into the "prehistory" filling mode by the current detector counters and waiting for the master pulse. For each event along with the measurement data, date, time, sequence number of the event in the monitoring session, and gain values of the ADC boards are recorded in the file. This service information is used to match the CDD events with events on large and small Cherenkov installations.

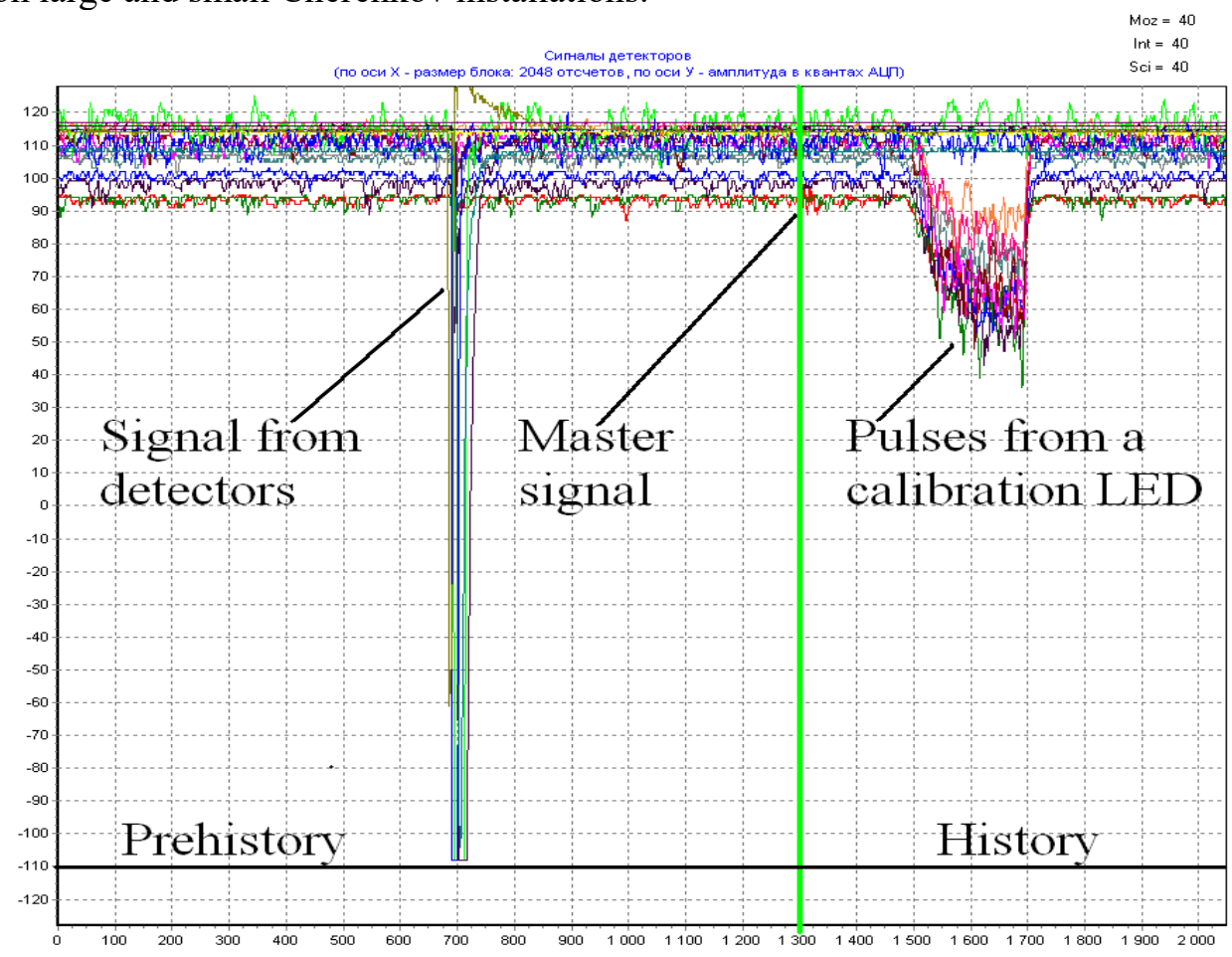

Figure 5: Recording of EAS event frame

Preliminary analysis includes viewing frames on a time-extended scale, finding a useful signal in the "prehistory" and determining the parameters of the signals - amplitude, area, halfwidth and maximum moment. During the observation session, several hundred events are recorded depending on the duration of the session (moon phases and weather conditions), a useful signal being present only in some of these events, because most of the events occur far from the CDD beyond the viewing angle and sensitivity of the detectors.

Calibration measurements of detectors make it possible to determine the signal level from one particle of the system "PMU - preamplifier - ADC" [9]. The detectors are calibrated 
alternately with the aid of a special plastic, which is mounted on the photocathode of the calibrated photomultiplier as the source of the known signal.

Several additional Cherenkov integral detectors, scintillation detectors, as well as one muon detector, operate at each monitoring station. In addition, an antenna for measuring EAS radio emission [10] (Fig. 3) was installed at the Obscural station and Wide field-of-view Cherenkov telescope for the detection of cosmic rays in coincidence with the Yakutsk extensive air shower array [11]

With the help of a complex of various detectors with different operation thresholds and registration type, fast ADCs with a sampling frequency of up to $250 \mathrm{MHz}$, it is possible to obtain detailed information on the structure and composition of the EAS disk on the observation plane.

\section{The algorithm used in the calibration of Cherenkov receivers of a laser burst in the atmosphere at a wavelength of $\square=532 \mathrm{~nm}$}

Since the spectral characteristic of the Cherenkov EAS light receiver lies in the wavelength region of 300-800 $\mathrm{nm}$, the selected monochromatic laser with $\lambda=532 \mathrm{~nm}$ and a sufficiently large output power can create a flash of light reaching up to stratospheric altitudes. This completely covers the area of the maximum illumination of the Cerenkov EAS light, and hence, makes it possible to control the receiver sensitivity.[12]
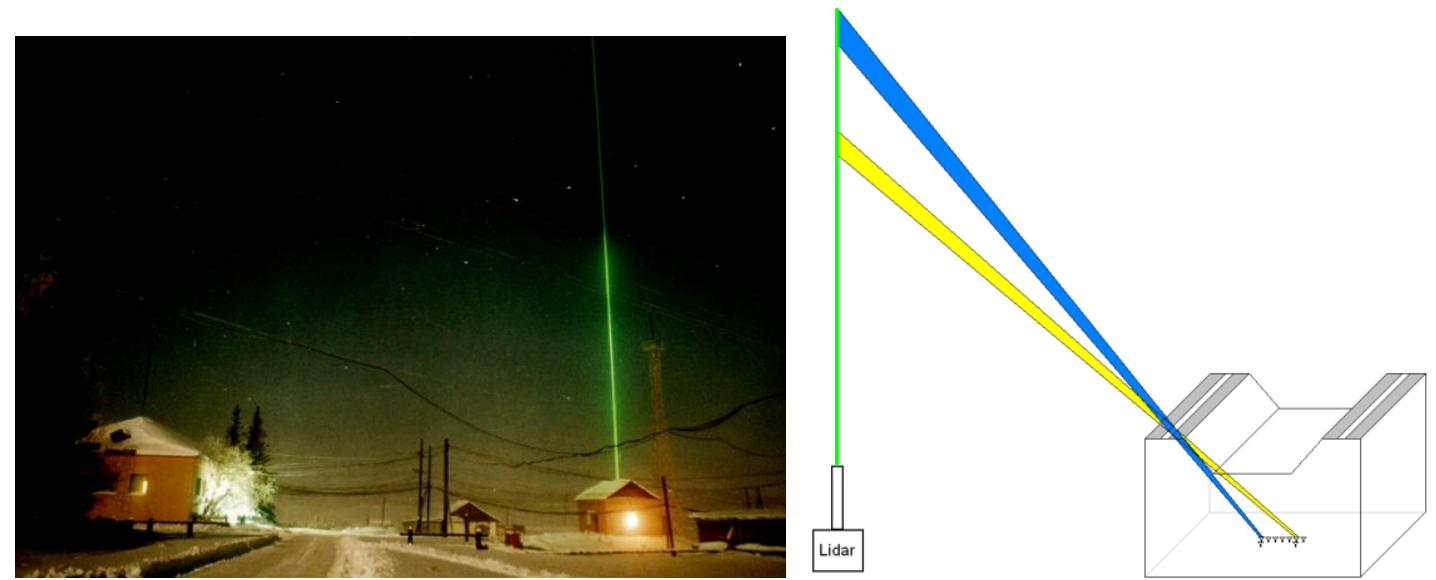

Figure 6: Diagram of Cherenkov detector calibration with the help of a laser "flash".

Differential detectors of the installation observe certain angles of the sky, and this circumstance makes it possible to calculate the number of photons hitting the photocathode of the photomultiplier. The response of each photomultiplier is related to a known number of photons generated by the laser. The calibration diagram is shown in Fig. 6.

Table 1: The range of visible photomultipliers of the lidar radiation levels for Obscura 1

\begin{tabular}{|l|l|l|l|l|l|l|l|l|l|l|}
\hline PMT No. & 1 & 2 & 3 & 4 & 5 & 6 & 7 & 8 & 9 & 10 \\
\hline Min. height, $\mathrm{m}$ & 1134 & 972 & 852 & 756 & 681 & 618 & 567 & 525 & 1047 & 546 \\
\hline $\begin{array}{l}\text { Max. height, } \\
\mathrm{m}\end{array}$ & 1455 & 1197 & 1020 & 885 & 885 & 702 & 639 & 582 & 1314 & 609 \\
\hline
\end{tabular}

With the help of the geometric method and by taking into account the aperture of Cherenkov receivers, we restored the heights observed by each of the photomultipliers (see Table1), and the parameters of the recorded pulses, including the pulse area, were attributed to these heights. In this case, the area of the pulse reflects the number of photons coming from a given height with known intensity. It is the constant intensity of laser radiation, and so the shape of the recorded pulse, which is the basis of the chosen calibration method. Change in weather condi- 
tions, i.e. the conditions for the propagation of optical signals in the atmosphere, requires the introduction of corrections in the analysis of the calibration data.

\section{Method of the Cherenkov channel calibration by means of a LIDAR and an optical radiator}

At the Yakutsk array, LIDAR measures the temperature of the upper layers and the transparency of the atmosphere [13]. The LIDAR research station is located near the center of the Yakutsk array (Fig. 1), which makes it possible to calibrate Cherenkov detectors without changing the output characteristics of the laser. In addition, LIDAR is used as a test of the operability of the optical part of the Cherenkov installation.

LIDAR is permanently installed in the center of the Yakutsk array and "shoots" strictly vertically to the installation plane. The control is carried out with the aid of a program of the small Cherenkov installation, after putting the laser into a given mode of generating radiation at a wavelength of $\lambda=530 \mathrm{~nm}$, which was required to put the laser into a mode with a given power and a photon flux known from the passport.

\section{Conclusion}

Currently, work is underway to modernize the Yakutsk extensive air shower array. The debugging of the new version of electronic equipment of observation stations and the method of data collection and processing. Increasing the accuracy of the station's timing synchronization. This will give us a significant improvement in the accuracy of determining the parameters of air showers, localization of the shower axis in space, i.e. coordinates of the shower axis in the installation plane, the zenith $\theta$ and the azimuth $\varphi$ angles.

It is planned to create its own trigger, which will allow us to determine the direction of the EAS arrival independently of the larger installation.

To increase the amount of the PMT photomosaic of the Cherenkov differential detector, which will give us an increase in the viewing angle of the detector and the accuracy of determining the shape of the cascade curve of the shower development in the atmosphere.

\section{Acknowledgements}

The work is supported by the program of Presidium of RAS "High-energy physics and neutrino astronomy" and by RFBR grant 16-29-13019 ofi-m.

The research was supported by "Scientific and Educational Foundation for Young Scientists of Republic of Sakha(Yakutia)" 20170220236

\section{References}

[1] J.V. Jelley Cherenkov Radiation and its Applications Pergamon Press, Oxford, New York (1958)B. Baggins, There and back again, Imladris Editions, Rivendell 3018.

[2] A.A. Watson Nuclear Physics B—Proceedings Supplements, 212-213 (2011), p. 13

[3] R. Mirzoyan Astroparticle Physics, 239-240 (2013), p. 26

[4] V.I. Zatsepin Journal of Experimental and Theoretical Physics, 47 (1964), p. 689

[5] Yu.A. Fomin, G.B. KhristiansenYadernaya Fizika, 14 (1971), p. 642

[6] Artamonov, V. P., Afanasiev, B. N., Glushkov, A. V. et al. Current state and prospects of the Yakut EAS array. Proceedings of the Russian Academy of Sciences, Phys. series, 1994, v. 58, No. 12, pp. 92-97

[7] Garipov, G.K. and Khrenov, B.A. // J.Phys.G: Nucl.Part. Phys., 20, 1981-1988, 1994. 
[8] Glushkov, A.V., Diminstein, O.S., Egorov, T.A. et al. Control and monitoring at the Yakutsk EAS array. // Experimental methods for studying cosmic rays of ultrahigh energies. - Yakutsk: YaF SB AS USSR, 1974, pp. 43-47

[9] Glushkov, A.V., Diminstein, O.S., Egorov, T.A. et al. Control and monitoring at the Yakutsk EAS array. // Experimental methods for studying cosmic rays of ultrahigh energies. - Yakutsk: YaF SB AS USSR, 1974, pp. 43-47

[10] S.P. Knurenko, Z.E. Petrov, I.S. Petrov. Radio emission of air showers with extremely high energy measured by the Yakutsk Radio Array. Nuclear Instruments and Methods in Physics Research A. 866 (2017) 230-241.

[11] A.A. Ivanov, S.P. Knurenko, A.D. Krasilnikov, Z.E. Petrov, M.I. Pravdin, I.Ye. Sleptsov, L.V. Timofeev. Wide field-of-view Cherenkov telescope for the detection of cosmic rays in coincidence with the Yakutsk extensive air shower array // Nuclear Instruments and Methods in Physics Research A 772 (2015) 34-42.

[12] S.P. Snurenko, Yu.A. Egorov, I. Petrov. Optic detectors calibration for measuring ultra-high energy extensive air showers Cherenkov radiation by $532 \mathrm{~nm}$ laser //Proc. SPIE 9292, 20th International Symposium on Atmospheric and Ocean Optics: Atmospheric Physics, 929258

[13] S.V. Nikolashkin, S.V. Titov. Wavelet analysis of wave processes in the Middle atmosphere over Yakutsk during the sudden stratospheric warming of major type in January, 2010 / Proc. SPIE 